\title{
ACCURACY OF VOXEL BASED SUPERIMPOSITION IN EVALUATION OF ORTHODONTIC CASES
}

\author{
Sahar Mohamed Samir" ${ }^{*}$ Walaa Mohamed Hamed ${ }^{* *}$, \\ Noha Hussein Abaas*** and Mary Medhat Farid**
}

\begin{abstract}
Aim: To compare the accuracy of 3D superimposed models produced by two programs using voxel based superimposition in follow up of orthodontic cases.

Patients and methods: The study included 21 non-growing patients performing orthodontic treatment. The gold standard was obtained by tracing each CBCT scan then recording linear measurements between evaluation points. T1 pre-treatment and $\mathrm{T} 2$ post-treatment scans were fused forming 3D superimposed model using two software programs; In Vivo (Anatomage, San Jose, California, USA), and Ondemand 3D (Ondemand 3D; Cybermed Co., Seoul, Korea). Accuracy as well as inter-observer reliability between three trained observers were calculated.
\end{abstract}

Results: Cronbach's alpha test showed significant low accuracy of superimposed models performed by In Vivo on canine and premolar readings and those obtained by Ondemand 3D showed significant low accuracy in canine readings. Cronbach's alpha test showed significant moderate inter-observer reliability in canine readings and significant low inter-observer reliability in molar readings performed by InVivo. While inter-observer reliability of on demand 3D was significantly moderate in molar and canine readings.

Conclusion: Statistical results showed low accuracy of 3D superimposed models performed by InVivo and Ondemand. In OnDemand showed higher inter observe reliability than InVivo.

KEYWORDS: Cone beam CT; Orthodontics; Computer guided 3D imaging

\section{INTRODUCTION}

Medical image registration/superimposition is the principle of bringing all the information from a given patient together into a single representation.
The application of medical image registration can be applied to images of the same kind (mono-modality) such as 2 CBCT images, or to images acquired by different techniques (multi-modality) such as registration of PET/CT images. Superimposition

\footnotetext{
* Ass. Lecturer of Oral and Maxillofacial Radiology Faculty of Dentistry Ain Shams University

** Ass. Professor of Oral and Maxillofacial Radiology Faculty of Dentistry Ain Shams University

*** Lecturer of Orthodontics Faculty of Dentistry Ain Shams University
} 
can be classified as point based, surface based or voxel based registration. (Maes et al., 2003)

Plane point registration (PPR) methods rely on manually detected anatomical landmarks. This method is labour-intensive if user interaction is required and its accuracy relies on the accurate localization of enough corresponding landmarks in all modalities. (Maes et al., 2003)

Surface based registration $(S B R)$ requires delineation of corresponding surfaces in each of the images separately. However, surface segmentation algorithms are generally highly data and application dependent and difficult to automate, and surfaces are not easily identified in functional modalities such as PET. (Maes et al., 2003)

Voxel based registration(VBR) methods measure the similarity of all geometrically corresponding voxel pairs. The main advantage is that feature calculation is straightforward when only greyvalues are used. (Maes et al.,2003) Voxel similarity algorithms are those used to measure the similarity between geometrically corresponding voxel pairs. Biomedical engineering described several types of VBR including; joint entropy, mutual information, and normalized mutual information. (Maes et al., 2003)

It is important to estimate the changes in tooth position before and after orthodontic treatment to determine whether the teeth moved to the planned direction and amount required to achieve. (Park et al., 2012)

Many articles assumed relative inaccuracy of different cephalometric superimposition methods and they claimed that none of the 2D superimposition methods is superior to the others. (Jacobson and Jacobson, 2006) This study aimed to assess the accuracy of two voxel based 3D superimposition software programs in evaluation of orthodontic cases.

\section{PATIENTS AND METHODS}

All procedures followed were approved by the research ethical committee and in accordance with the ethical standard. The detailed steps of the procedure were fully explained to the patient in a comfortable setting and she signed an informed consent. The research was based on pre-treatment (T1) and post-treatment (T2) CBCT DICOM images of 21 patients from the orthodontic clinic of Faculty of Dentistry Ain Shams University.

\section{CBCT Data}

All CBCT scans were acquired using I-CAT (Imaging Sciences International, Hatfield, Pennsylvania, USA) with voxel size $0.3 \mathrm{~mm}$, exposure cycle of $17.8 \mathrm{~s}$ and KVP of 120 . The field of view (FOV) was $23 \mathrm{~cm}$ diameter and $17 \mathrm{~cm}$ height. Pre-treatment (T1) scan was obtained before start of expansion while post-treatment (T2) scan was obtained 6 months after active expansion (retention period in which the expander is kept passive in place).

All DICOM files were transferred to the data base of the following 3rd party software programs; In Vivo (Anatomage, San Jose, California, USA), and Ondemand 3D (Ondemand 3D; Cybermed Co., Seoul, Korea).

\section{Calculation of the gold standard}

The gold standard was calculated from 3D analysis of each CBCT image (T1 and T2) using InVivoDental (Anatomage, San Jose, CA, USA) after setting the coordinates system for analysis using Frankfort horizontal plane and mid sagittal plane perpendicular to it. Then the points of evaluation were measured automatically from the mid sagittal plane.

The Frankfort horizontal plane was set by tracing of Porion (upper margin of external auditory meatus) and Orbitale (the lowest point in the margin of the orbit) points bilaterally then the software automatically set the plane and oriented 
the image according to this position. Setting mid sagittal plane was done by tracing of Nasion (Na: the fronto nasal suture) and Basion (Ba: the most anterior point of foramen magnum) points then the program automatically located the mid sagittal plane perpendicular to the Frankfort horizontal plane and adjusted the coordinate system for analysis.

Measurements were performed by tracing of each point after that the software automatically calculates its distance from midsagittal plane. Points of assessment were; the mid buccal point of canine, $2^{\text {nd }}$ premolar and $1^{\text {st }}$ molar bilaterally at the axial level of anterior cemento-enamel junction (CEJ). Mathematical calculation of the resulting measurements of T1 and T2 traced points of assessment were calculated manually and tabulated in an excel sheet to act as a gold standard (Figure 1).

\section{D superimposition using InVivoDental software}

InVivoDental (Anatomage, San Jose, CA, USA) version 5.2 was used at the work station of the specialized imaging center. The selected DICOM volume was opened then the "superimposition" tab was selected to open the superimposition tool bar. "Volume Registration" icon from the Toolbar was selected to open the Volume Registration interface. Volume of Interest "VOI" was selected by Leftclick on any of the 2D cross-sections to place the center of the VOI at the cranial base. Drawing of VOI Box was set to the default measurements ( $x$, $\mathrm{y}, \mathrm{z})=50 \mathrm{~mm} \times 50 \mathrm{~mm} \times 40 \mathrm{~mm}$. After finishing the superimposition, the layout was adjusted to the axial section at the level of CEJ of anterior teeth to start measurements at the points of assessment. Finally, the data of superimposition was saved using the "Save Superimposition" button (Figure 2).

\section{$3 D$ superimposition using On Demand 3D soft- ware}

On Demand 3D (CyberMed Inc., Seoul, South Korea) version 1.0.10.5385 was used at the specialized imaging centre's work station. The patient name was selected from Master data base "DBM" from the module bar at the left column of the OnDemand3D ${ }^{\mathrm{TM}}$ layout. To launch the "fusion" module, the two series of data (T1 and T2 images) were selected at once using the [CTRL] or [Shift] keys from DBM. The two sets of data from the "Loading Option" dialog were selected again using either "Shift" or "Select All" at once then "OK". The pre-operative DICOM data T1 was set as the adjustable one (secondary) and the post-operative T2 was set as the primary or the fixed scan. The region of interest was adjusted to the anterior cranial base using "VOI" icon.

"Manual Registration" tab was selected first before automatic registration. The secondary image was manipulated by Click and drag to re-position it close to the primary image. "Auto Registration" module was then clicked to start voxel based registration. The viewing tools and the color scale were adjusted to get the best image quality. Direct measurement of the points of assessment were done on the axial cut at the level of CEJ of anterior teeth using (the ruler) tool. The results were finally saved as projects using (save project tool) (Figure 3).

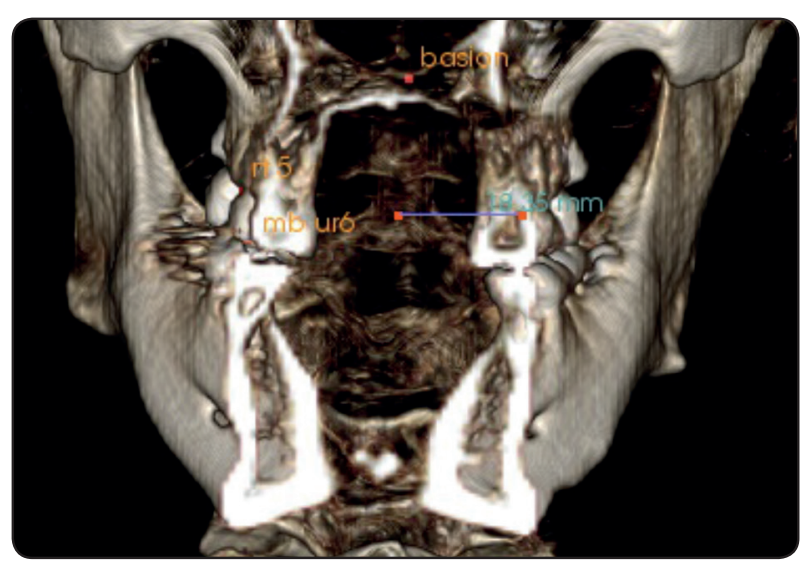

Fig. (1) Measuring the distance at mid buccal surface of left 2nd premolar at the level of anterior CEJ from the midsagittal plane. The 3D image was cropped coronally to the level of 2 nd premolar 


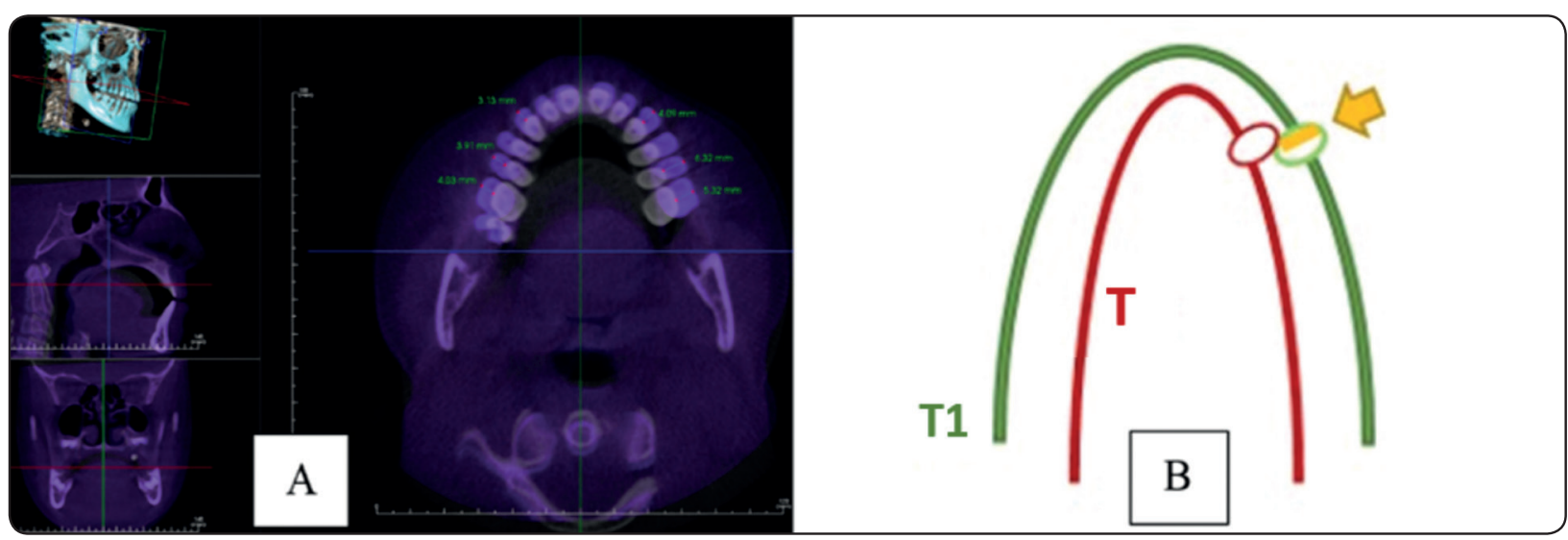

Fig. (2) A: The distance change on the axial cut at the level of CEJ of anterior teeth using VBR of InVivo dental. The distance was measured from mid buccal point of $\mathrm{T}$ to mid buccal point of $\mathrm{T} 1$ parallel to their long axis. B: diagrammatic representation of superimposed T-T1 model. The distance change of canine measured directly from mid buccal surfaces of canine at $\mathrm{T}$ and canine at $\mathrm{T} 1$ passing with the long axis.

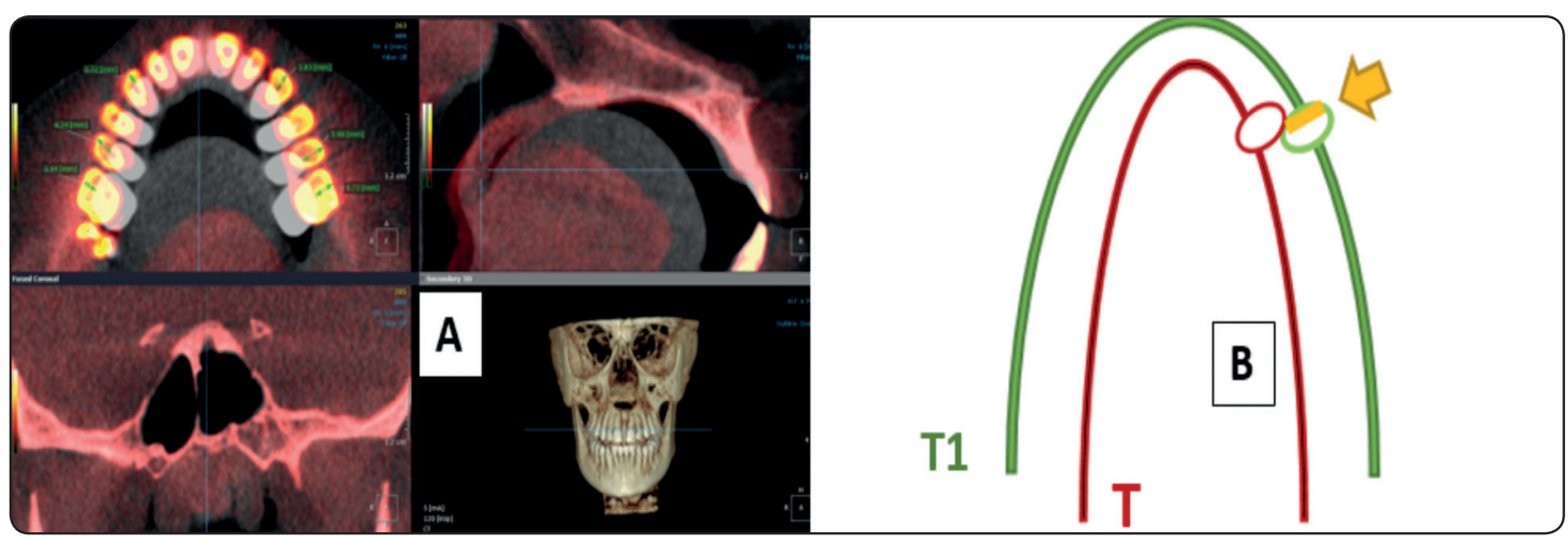

Fig. (3) A: The distance change on the axial cut at the level of CEJ of anterior teeth using VBR of OnDemand 3D. The distance was measured from mid buccal point of $\mathrm{T}$ to mid buccal point of $\mathrm{T} 1$ parallel to their long axis (note the fused volume at the cranial base) B: diagrammatic representation of superimposed T-T1 model. The distance change of canine measured directly from mid buccal surfaces of canine at $\mathrm{T}$ and canine at $\mathrm{T} 1$ passing with the long axis.

\section{RESULTS}

Cronbach's alpha test was used to test :

a. Accuracy of each method compared with the gold standard

b. Inter-observer reliability between 3 observers regarding 3 points; canine, premolar and molar on the $3 \mathrm{D}$ superimposed models of the two software programs

Cronbach's alpha test showed significant low accuracy of superimposed models performed by In Vivo on canine and premolar readings and those obtained by Ondemand 3D showed significant low accuracy in canine readings. Cronbach's alpha test showed significant moderate inter-observer reliability in canine readings and significant low inter-observer reliability in molar readings performed by InVivo. While inter-observer reliability of on demand 3D was significantly moderate in molar and canine readings. 
TABLE (1) Accuracy in canine readings:

\begin{tabular}{|c|c|c|c|}
\hline Observer & Method & CA (95\% CI) & P \\
\hline $\mathbf{1}$ & InVivo & $0.506(0.032-0.794)$ & 0.146 \\
\hline $\mathbf{2}$ & OnDemand & $0.271(-0.243-0.666)$ & $\mathbf{0 . 0 0 5 *}$ \\
\hline & InVivo & $0.609(0.180-0.844)$ & $\mathbf{0 . 0 2 0} *$ \\
\hline $\mathbf{3}$ & OnDemand & $0.504(0.029-0.793)$ & 0.477 \\
\hline ALL obsevers & InVivo & $0.015(-0.470-0.494)$ & 0.194 \\
\hline & OnDemand & $0.224(-0.290-0.637)$ & $0.008^{*}$ \\
\hline
\end{tabular}

CA: Cronbach's Alpha, *Significant, CI: Confidence interval

TABLE (2) Accuracy in premolar readings:

\begin{tabular}{|c|c|c|c|}
\hline Observer & Method & CA $(\mathbf{9 5} \%$ CI $)$ & P \\
\hline $\mathbf{1}$ & InVivo & $0.661(0.262-0.867)$ & 0.210 \\
\hline $\mathbf{2}$ & OnDemand & $0.209(-0.303-0.628)$ & $\mathbf{0 . 0 1 7} *$ \\
\hline & InVivo & $0.518(0.048-0.800)$ & 0.063 \\
\hline $\mathbf{3}$ & OnDemand & $0.386(-0.118-0.732)$ & 0.945 \\
\hline ALL observers & InVivo & $-0.509(-0.851-.134)$ & 0.520 \\
\hline & OnDemand & $-0.013(-0.492-.472)$ & $\underline{\mathbf{0 . 0 1 6 *}}$ \\
\hline
\end{tabular}

CA: Cronbach's Alpha, *Significant, CI: Confidence interval

Table (3): Accuracy in molar readings:

\begin{tabular}{|c|c|c|c|}
\hline Observer & Method & CA $(\mathbf{9 5} \%$ CI $)$ & P \\
\hline $\mathbf{1}$ & InVivo & $0.511(0.038-0.797)$ & 0.057 \\
\hline $\mathbf{2}$ & OnDemand & $0.397(-0.106-0.738)$ & $\mathbf{0 . 0 0 5 *}$ \\
\hline & InVivo & $0.603(0.170-0.841)$ & 0.226 \\
\hline $\mathbf{3}$ & OnDemand & $0.195(-0.316-0.619)$ & 0.995 \\
\hline ALL observers & InVivo & $-0.603(-0.841--0.170)$ & 0.956 \\
\hline & OnDemand & $-0.425(-0.753-0.072)$ & 0.103 \\
\hline
\end{tabular}

CA: Cronbach's Alpha, *Significant, CI: Confidence interval 
TABLE (4) Inter-observer reliability:

\begin{tabular}{|c|c|c|c|c|c|c|}
\hline & \multicolumn{2}{|c|}{ Canine } & \multicolumn{2}{c|}{ Premolar } & \multicolumn{2}{c|}{ Molar } \\
\hline & CA & P & CA & P & CA & P \\
\hline In Vivo & $\begin{array}{c}0.531 \\
(0.207-0.736)\end{array}$ & $<0.001^{*}$ & $\begin{array}{c}0.227 \\
(-0.448-0.617)\end{array}$ & 0.208 & $\begin{array}{c}0.430 \\
(0.037-0.680)\end{array}$ & $0.018^{*}$ \\
\hline $\begin{array}{c}\text { On } \\
\text { Demand }\end{array}$ & $\begin{array}{c}0.528 \\
(0.202-0.735)\end{array}$ & $<0.001^{*}$ & $\begin{array}{c}0.308 \\
(-0.169-0.611)\end{array}$ & 0.084 & $\begin{array}{c}0.696 \\
(0.487-0.829)\end{array}$ & $<0.001^{*}$ \\
\hline
\end{tabular}

\section{DISCUSSION}

Nowadays, the advancement of CBCT imaging directed the orthodontic analysis towards 3D imaging. Various third party software programs are now providing features for CBCT superimposition of serial images. Nonetheless, there is still a deficiency in comprehensive studies about 3D superimposition software programs also there are so many controversial issues and unanswered questions regarding this process. This study was designed to compare the accuracy of two voxel based software programs.

Each candidate in this study has been scanned twice at (T) then (T1). Dental expansion outcome can be evaluated at T0 (immediately after expansion), T1 (short term) after six month or T2 (long term) after 12 month Lagravere et al., 2010. In this study, we evaluated short term dental changes after six months that was the retention period where the expander is kept passive in place after active expansion.

All scans were acquired using the iCAT 3D Imaging System (Imaging Sciences International Inc, Hatfield, PA, USA) using a standardized imaging protocol for all CBCT scans. Axial cut (Transverse section) was the plane of assessment in our study based on Lagravere et al., 2010 who explained that maxillary expansion results in dental effects, greater than skeletal one and the changes are almost on transverse direction.
In the current study, the whole Cranial base was selected as a stable unchanged landmark for VBR as recommended by Cevedens et al., 2006 who described the cranial base as a stable area for non-growing cases and the anterior cranial base for growing patients. Nada et al., 2011 compared VBR methods on anterior cranial base with those on Zygomatic arch by Maxillim (Medicim NV, Mechelen, Belgium) software and concluded that both registration areas were accepted. Likewise, VBR was performed on cranial base by Motta et al., 2010 using Imagine software in evaluation of orthognathic surgical outcome. Moreover, Koerich et al., 2016 described VBR as a perfect superimposition method when cranial base structures are used as a fixed area for registration.

The documented results for InVivo volume (VBR) and OnDemand (VBR) in this research were; low accuracy and moderate to low interobserver reliability. Changes resulting from dental maxillary expansion unilaterally in our work were less than $4 \mathrm{~mm}$. Accordingly, $0.5 \mathrm{~mm}$ disagreement between observers will result in significant statistical difference, yet, in clinical practice it is a neglectable difference. The results were attributed to the small variable under investigation and we are recommending re-examination of those methods using larger variables such as those resulting from surgical interventions. 
Several studies such as park et al., 2012 and Nada et al., 2011 reported accurate results for VBR however, they didn't provide a gold standard for their evaluation.

Regarding Inter-observer reliability onDemand showed better results than inVivo. Yet, both software had moderate to low reliability. Similarly, Cevedens et al., 2009 used Imagine software VBR for registration of segmented surface models of growing cases then reported similar inter-examiner range of variation. Koriesh et al., 2016 as well used maxillary and mandibular VBR using OnDemand then reported excellent reproducibility of the results. Almokhtar et al., 2014 compared VBR to SBR and reported lower variability with VBR.

Analysis of 3D change on 2D axial cuts is a main limitation of this work. Recent upgrades allow 3D analysis with colour coded distance map.

The authors declare no conflict of interest regarding the used software program.

\section{CONCLUSION}

Statistical results showed low accuracy of 3D superimposed models performed by InVivo and Ondemand. In OnDemand showed higher inter observe reliability than InVivo.

\section{REFERENCES}

1. Almukhtar A, Ju X, Khambay B, McDonald J, Ayoub A. Comparison of the accuracy of voxel based registration and surface based registration for 3D assessment of surgical change following orthognathic surgery. PloS one. 2014 Apr 2;9(4):e93402.

2. Cevidanes LH, Bailey LJ, Tucker GR, Styner MA, Mol A, Phillips CL, Proffit WR, Turvey T. Superimposition of 3D cone-beam CT models of orthognathic surgery patients.
Dento maxillo facial radiology. 2005 Nov;34(6):369-75.

3. Cevidanes LH, Heymann G, Cornelis MA, DeClerck HJ, Tulloch JC. Superimposition of 3-dimensional cone-beam computed tomography models of growing patients. American Journal of Orthodontics and Dentofacial Orthopedics. 2009 Jul 31;136(1):94-9.

4. Jacobson A, Jacobson RL, Khoury F, Antoun H, Missika P, Sclar AG. Radiographic Cephalometry: From Basics to 3-D Imaging, (Book/CD-ROM set). 2007 Nov.

5. Koerich L, Burns D, Weissheimer A, Claus JD. Threedimensional maxillary and mandibular regional superimposition using cone beam computed tomography: a validation study. International journal of oral and maxillofacial surgery. 2016 May 31;45(5):662-9.

6. Lagravère MO, Major PW, Carey J. Sensitivity analysis for plane orientation in three-dimensional cephalometric analysis based on superimposition of serial cone beam computed tomography images. Dento maxillo facial radiology. 2010 Oct;39(7):400-8.

7. Maes F, Vandermeulen D, Suetens P. Medical image registration using mutual information. Proceedings of the IEEE. 2003 Oct;91(10):1699-722.

8. Motta AT, Carvalho FD, Oliveira AE, Cevidanes LH, Almeida MA. Superimposition of 3D cone-beam CT models in orthognathic surgery. Dental press journal of orthodontics. 2010 Apr;15(2):39-41.

9. Nada RM, Maal TJ, Breuning KH, Berge SJ, Mostafa YA, Kuijpers-Jagtman AM. Accuracy and reproducibility of voxel based superimposition of cone beam computed tomography models on the anterior cranial base and the zygomatic arches. PLoS One. 2011 Feb 9;6(2):e16520.

10. Park TJ, Lee SH, Lee KS. A method for mandibular dental arch superimposition using 3D cone beam CT and orthodontic 3D digital model. The Korean Journal of Orthodontics. 2012 Aug 1;42(4):169-81.

11. Zamora N, Llamas JM, Cibrián R, Gandia JL, Paredes V. Cephalometric measurements from 3D reconstructed images compared with conventional 2D images. The Angle Orthodontist. 2011 Apr 7;81(5):856-64. 